\title{
Real-Time Nonlinear Model Predictive Controller for Multiple Degrees of Freedom Wave Energy Converters with Non-Ideal Power Take-Off
}

\author{
Ali S. Haider ${ }^{1} *$ D, Ted K. A. Brekken ${ }^{1}$ and Alan McCall ${ }^{2}$ \\ 1 Electrical Engineering and Computer Science, Oregon State University, Corvallis, OR 97331, USA; \\ brekken@eecs.oregonstate.edu \\ 2 Dehlsen Associates, LLC., Santa Barbara, CA 93101, USA; amccall@ecomerittech.com \\ * Correspondence: haidera@oregonstate.edu
}

check for updates

Citation: Haider, A.S.; Brekken, T.K.A.; McCall, A. Real-Time Nonlinear Model Predictive Controller for Multiple Degrees of Freedom Wave Energy Converters with Non-Ideal Power Take-Off. J. Mar. Sci. Eng. 2021, 9, 890. https:// doi.org/10.3390/jmse9080890

Academic Editor: Constantine Michailides

Received: 3 August 2021

Accepted: 16 August 2021

Published: 18 August 2021

Publisher's Note: MDPI stays neutral with regard to jurisdictional claims in published maps and institutional affiliations.

Copyright: (c) 2021 by the authors. Licensee MDPI, Basel, Switzerland. This article is an open access article distributed under the terms and conditions of the Creative Commons Attribution (CC BY) license (https:/ / creativecommons.org/licenses/by/ $4.0 /)$.

\begin{abstract}
An increase in wave energy converter (WEC) efficiency requires not only consideration of the nonlinear effects in the WEC dynamics and the power take-off (PTO) mechanisms, but also more integrated treatment of the whole system, i.e., the buoy dynamics, the PTO system, and the control strategy. It results in an optimization formulation that has a nonquadratic and nonstandard cost functional. This article presents the application of real-time nonlinear model predictive controller (NMPC) to two degrees of freedom point absorber type WEC with highly nonlinear PTO characteristics. The nonlinear effects, such as the fluid viscous drag, are also included in the plant dynamics. The controller is implemented on a real-time target machine, and the WEC device is emulated in real-time using the WECSIM toolbox. The results for the successful performance of the design are presented for irregular waves under linear and nonlinear hydrodynamic conditions.
\end{abstract}

Keywords: nonlinear model predictive control; two degrees of freedom wave energy converter; nonlinear hydrodynamics; nonlinear power take-off

\section{Introduction}

Renewable energy technologies present a viable and sustainable contribution to the world's growing energy demands, and the ocean provides potential for an enormous untapped energy resource for the world's energy portfolio [1,2]. The prospect of ocean wave energy has triggered research in optimal power capture techniques for wave energy converters, including non-ideal operating conditions, such as the non-ideal PTO system constraints [3] and nonlinear sea conditions. Achieving optimal power capture by a WEC in practice is a multifaceted objective. It depends on various factors, such as the physical design of the WEC, the design of the PTO system, the ocean conditions, and the control techniques.

Model predictive control (MPC) is a promising control approach for wave energy converters' relatively slow plant dynamics because it maximizes energy capture while respecting the system's mechanical limits. MPC is a look-ahead control strategy that predicts future system behavior to solve a constrained optimization problem and determines the best control action to maximize the output power of WEC. MPC and other optimal control schemes, such as pseudospectral methods and MPC-like algorithms, have been comprehensively studied in the literature for a single WEC device and an array of wave energy converters [4-9]. An MPC algorithm uses an internal model of the plant to predict the system's future states [10]. Nonlinear control algorithms can consider the non-ideal operating conditions and nonlinear effects, including but not limited to non-ideal power take-off mechanism [11], nonlinear viscous drag terms [12,13], and nonlinear mooring dynamics [2]. The non-ideality of PTO systems in most literature is limited to the efficiency of the PTO mechanism [13-16]. One of the motivations for this research is to consider 
higher-order nonlinear PTO characteristics as an optimization objective for the NMPC problem. The economic MPC techniques consider a general economic cost function directly in real time [17-19]. However, we have deployed a real-time iterative (RTI) algorithm [20,21] to optimize a more general class of non-ideal PTO mechanisms using pseudo-quadratic formulations [3]; this method also supports nonlinearities in the plant dynamics, such as mooring and fluid viscous drag. Another motivation for this work is investigating nonlinear multiple degrees of freedom WEC coupled to non-ideal PTO.

Lots of work has been focused on studying multiple degrees of freedom WEC devices that prove a significant improvement of power capture by the WEC device. Multi-resonant feedback control of a three degree of freedom WEC is presented [22], where a linear hydrodynamic model is considered, and multi-resonant proportional-derivative control law is proposed where the focus is linear plant dynamics under unconstrained control. An analysis of a multi-degree-of-freedom point absorber WEC in the surge, heave, and pitch directions is presented in [23], and frequency and time-domain formulations are presented for the linear plant dynamics. A time-domain model for a point absorber WEC in six degrees of freedom is developed in [24] with an optimal resistive loading. The three degrees of a freedom model of a WEC is presented in [25], where the capture performance of various PTO systems is investigated for a linear plant model. An active control strategy based on the optimal velocity trajectory tracking for a multi-DoF submerged point absorber WEC is presented in [26], where a linearized dynamic system model is considered along with an ideal PTO mechanism. A nonlinear MPC design and implementation based on differential flatness parameterization has been proposed in [27]. Given that most of the work focused on linear plant dynamics for multiple degrees of freedom WEC or ideal PTO mechanisms, and the lack of application of NMPC for such class or problems, we have investigated the application of NMPC to nonlinear multiple DoF WEC plant with a non-ideal PTO mechanism, and focus on the real-time implementation of the control algorithm on a real-time target machine.

This research presents the maximization of power extraction by a 2-DoF WEC device, a WECSIM [28] model of the full-scale version of the Dehlsen Associates, LLC multi-pod CENTIPOD [29]. Although the CENTIPOD device is an array device, the cross-coupling between pods is ignored for this study, which is negligible for the sea conditions of interest in this work and will be investigated in the future. The goal is to optimize the power extracted by the heave and pitch PTOs subject to actuation and velocity constraints. The objective function is a nonstandard and nonquadratic functional of PTO force and velocity, resulting from a practical PTO generator power loss characteristic. The WEC model includes nonlinear viscous drag terms; hence, the resulting plant model is a nonlinear dynamic system. We have implemented an NMPC for the problem. To tackle a free-formed objective function subjected to nonlinear system dynamics, we have used the extended version of the NMPC design from [30], based on pseudo-quadratization using an ACADO toolkit [21]. No prior knowledge of wave excitation is assumed. The WEC model is simulated on a real-time emulator machine, while control is deployed on a Speedgoat real-time performance machine [31], which is interfaced with the WEC emulator machine through an ethernet port. The simulation results for real-time NMPC are presented for the linear and nonlinear hydrodynamics conditions simulated in WECSIM.

\section{Time Domain Model of a Multiple Degree of Freedom WEC}

The WEC device is a full-scale version of the Dehlsen Associates, LLC multi-pod CENTIPOD [29,32]. A 1:35-scale version of the device is shown in Figure 1. This CENTIPOD device has three floating pods and three spars fixed to a backbone structure. The backbone is anchored using mooring lines, as shown in Figure 2. In its 2-DoF version, each pod is attached to a PTO mechanism in the heave and pitch degrees of freedom. All pods in Figure 2 are assumed identical, and since the CENTIPOD device is an array device, the array effect [33] could become prominent as the significant height of the waves increases and the incident angle of the waves is not parallel to the $x$-axis in Figure 2. For this study, 
incident waves are assumed parallel to the $x$-axis, and for the sea state of interest in this work, the cross-coupling between the pods is very small and is neglected, although it will be investigated in future work.

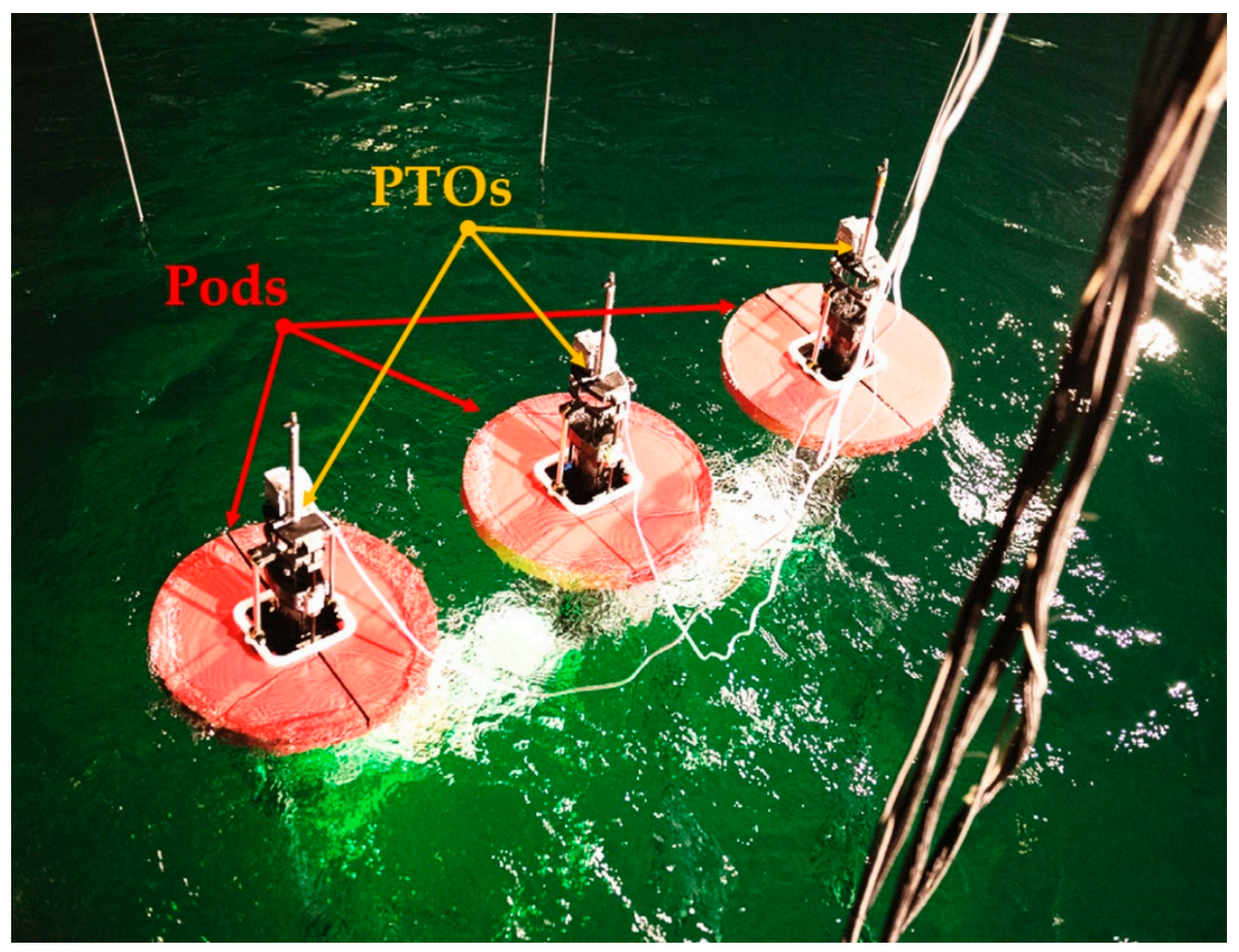

Figure 1. Image of the Dehlsen Associates, LLC, 1:35-scale CENTIPOD WEC.

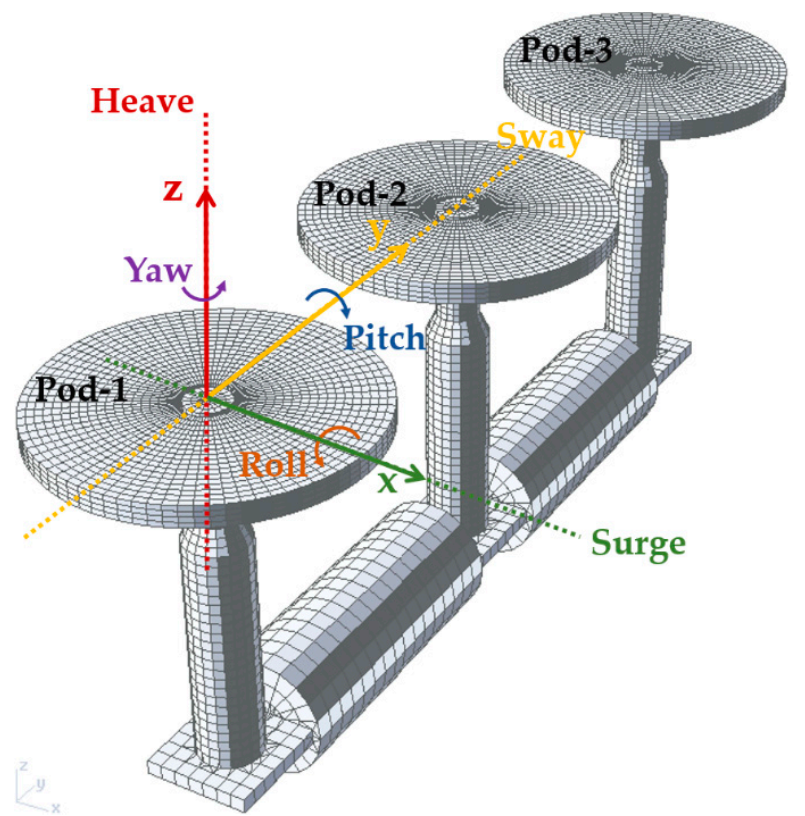

(a)

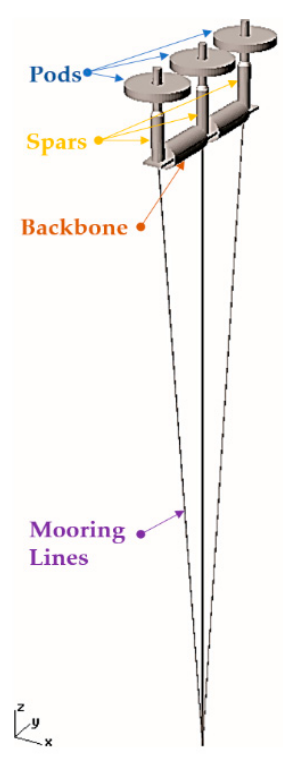

(b)

Figure 2. Degrees of freedom for dynamic modeling of CENTIPOD WEC: (a) baseline configuration; (b) model with mooring lines. 
We will follow the subscript notation of the WEC-Sim toolbox [28] for the degrees of freedom for WEC, in which the integers from $1,2, \ldots 6$ correspond to surge, sway, heave, roll, pitch, and yaw, respectively. Some other notations and symbols for WEC modeling are given in Table 1.

Table 1. Notations and symbols for WEC modeling.

\begin{tabular}{|c|c|}
\hline Variable & Description \\
\hline$v_{i}$ & Velocity (Linear or Angular) in $i^{\text {th }} \mathrm{DoF}$ \\
\hline$x_{i}$ & Displacement (Linear or Angular) in $i^{\text {th }} \mathrm{DoF}$ \\
\hline$\xi_{i}$ & Intermediate State variables for radiation force State-Space approximation \\
\hline$F_{r, p q}$ & Radiation force in $p^{\text {th }}$ DoF due to velocity in $q^{\text {th }} \mathrm{DoF}$ \\
\hline$F_{h s, i}$ & Hydrostatic force in $i^{\text {th }}$ DoF \\
\hline$F_{v, i}$ & Viscous drag force in $i^{t h}$ DoF \\
\hline$F_{e, i}$ & Wave excitation force in $i^{\text {th }} \mathrm{DoF}$ \\
\hline$F_{p, i}$ & PTO force in $i^{\text {th }} \mathrm{DoF}$ \\
\hline$m$ & Mass of the float \\
\hline$A_{p q}(\infty)$ & Added mass at the infinite frequency in $p^{\text {th }}$ DoF due to acceleration in $q^{\text {th }}$ DoF \\
\hline$C_{i}$ & The hydrostatic restoring coefficient in $i^{\text {th }}$ DoF \\
\hline$C_{v d, i}$ & Viscous drag coefficient in $i^{\text {th }}$ DoF \\
\hline$A_{q p}$ & Frequency-dependent added mass in $p^{\text {th }}$ DoF due to acceleration in $q^{\text {th }}$ DoF \\
\hline$B_{q p}$ & Frequency-dependent damping in $p^{\text {th }}$ DoF due to velocity in $q^{\text {th }}$ DoF \\
\hline$K_{p q}^{q p}$ & Radiation force impulse response without infinite frequency added mass \\
\hline$Z_{q p}^{p q}$ & WEC Intrinsic impedance response in $p^{\text {th }}$ DoF due to velocity in $q^{\text {th }}$ DoF \\
\hline$a_{i}$ & Polynomial coefficients \\
\hline$c_{i, j}$ & Polynomial coefficients for cost functional \\
\hline$I_{p, i}$ & $i^{\text {th }}$ PTO current \\
\hline$\eta_{\text {Conv }}$ & PTO converter efficiency \\
\hline$K_{C u}$ & PTO generator copper loss constant \\
\hline$R_{\Omega}$ & PTO generator winding resistance \\
\hline
\end{tabular}

\subsection{Surge-Pitch-Heave Model of WEC Modeling in State-Space Form}

Each pod in Figure 2 is modeling as a wave point absorber device. The Cummins equation for the coupled surge and pitch dynamics for a point absorber pod (assuming a local reference frame) is given by,

$$
\left(m+A_{11}(\infty)\right) \dot{v}_{1}+A_{15}(\infty) \dot{v}_{5}=-F_{r, 11}(t)-F_{r, 15}(t)-F_{v, 1}(t)+F_{e, 1}(t),
$$

$$
\left(m+A_{55}(\infty)\right) \dot{v}_{5}+A_{51}(\infty) \dot{v}_{1}=-F_{r, 55}(t)-F_{r, 51}(t)-F_{v, 5}(t)-F_{h s, 5}(t)-F_{p, 5}(t)+F_{e, 5}(t) .
$$

The Cummins equation for the heave dynamics of a point absorber pod is given by,

$$
\left(m+A_{33}(\infty)\right) \dot{v}_{3}(t)=-F_{r, 33}(t)-F_{h s, 3}(t)-F_{v, 3}(t)-F_{p, 3}(t)+F_{e, 3}(t),
$$

The hydrostatic, viscous damping, and radiation force terms in (1) through (3) are given by,

$$
\begin{gathered}
F_{r, i j}(t)=\int_{-\infty}^{t} K_{i j}(t-\tau) v_{j} d \tau, \\
F_{h s, i}(t)=C_{i} x_{i}, \\
F_{v, i}(t)=C_{d, i} v_{i}\left|v_{i}\right| .
\end{gathered}
$$

A transfer function expression can approximate the convolution integral term in (4),

$$
F_{r, p q}(t)=\int_{-\infty}^{t} K_{p q}(t-\tau) v_{q} d \tau \Leftrightarrow F_{r, p q}(j \omega)=Z_{p q}(j \omega) V_{q}(j \omega),
$$


Using the device data from WAMIT [34], we can approximate the intrinsic impedance $Z_{p q}(j \omega)$ in (7) by a second order transfer function using system identification techniques,

$$
Z_{p q}(j \omega)=\left.\left[j \omega\left(A_{p q}(j \omega)-A_{p q}(\infty)\right)+B_{q p}(j \omega)\right] \approx \frac{\alpha_{p q, 1} s+\alpha_{p q, 0}}{s^{2}+\beta_{p q, 1} s+\beta_{p q, 0}}\right|_{s=j \omega},
$$

Using (8) in (7) enables us to express the radiation force as a second-order transfer function,

$$
F_{r, p q}(s) \approx \frac{\alpha_{p q, 1} s+\alpha_{p q, 0}}{s^{2}+\beta_{p q, 1}+\beta_{p q, 0}} V_{q}(s),
$$

The transfer function expression in (9) can be converted to the state-space expressions in the observer canonical forms for each of the radiation force terms,

$$
\begin{gathered}
{\left[\begin{array}{c}
\dot{\xi}_{k}(t) \\
\dot{\xi}_{k+1}(t)
\end{array}\right]=\left[\begin{array}{cc}
0 & 1 \\
a_{k} & a_{k+1}
\end{array}\right]\left[\begin{array}{c}
\xi_{k}(t) \\
\xi_{k+1}(t)
\end{array}\right]+\left[\begin{array}{c}
b_{k} \\
b_{k+1}
\end{array}\right] v_{q}(t),} \\
y_{p q}(t)=\left[\begin{array}{ll}
1 & 0
\end{array}\right]\left[\begin{array}{c}
\xi_{k}(t) \\
\xi_{k+1}(t)
\end{array}\right] \approx F_{r, p q}(t) .
\end{gathered}
$$

By the comparison of (9)-(11), we have, $\alpha_{p q, 1}=b_{k}, \beta_{p q, 1}=-a_{k+1}, \beta_{p q, 0}=-a_{k}$, and $\alpha_{p q, 0}=b_{k+1}-b_{k} a_{k+1}$. Making a change of variables in (1),

$$
\begin{gathered}
M_{i i}=\left(m+A_{i i}(\infty)\right), \\
F_{1, \text { net }}=-F_{r, 11}(t)-F_{r, 15}(t)-F_{v, 1}(t)+F_{e, 1}(t), \\
F_{5, \text { net }}=-F_{r, 55}(t)-F_{r, 51}(t)-C_{5} x_{5}-F_{v, 5}(t)-F_{p, 5}(t)+F_{e, 5}(t) .
\end{gathered}
$$

Using (12)-(14) in (1), we get the pitch-surge coupled model of a pod as,

$$
\left[\begin{array}{l}
\dot{v}_{1} \\
\dot{v}_{5}
\end{array}\right]=\left[\begin{array}{cc}
M_{11} & A_{15}(\infty) \\
A_{51}(\infty) & M_{55}
\end{array}\right]^{-1}\left[\begin{array}{c}
F_{1, \text { net }} \\
F_{5, \text { net }}
\end{array}\right]
$$

The viscous drag force term $v_{i}\left|v_{i}\right|$ in (6) is a hard nonlinearity that may lead to convergence issues for the optimization solvers. One solution is to approximate this term with a soft nonlinearity by replacing it with a smooth higher-order polynomial. A third-order polynomial approximation for $v_{i}\left|v_{i}\right|$ is used in the surge and heave direction, where the range of interest of velocity is $v_{i} \in(-1.5,1.5) \mathrm{m} / \mathrm{sec}$, and a fifth-order polynomial approximation is used for pitch direction, where the range of interest of velocity is $v_{i} \in(-0.5,0.5) \mathrm{rad} / \mathrm{sec}$. With, $p_{i, j}$ being the $j^{t h}$ polynomial coefficient for $i^{\text {th }}$ degree polynomial curve fit

$$
\begin{aligned}
& F_{v, i}=C_{d, i} v_{i}\left|v_{i}\right| \approx C_{d, i}\left(p_{3,3} v_{i}^{3}+p_{3,1} v_{i}\right), \quad i=1,3, \\
& F_{v, 5}=C_{d, 5} v_{5}\left|v_{5}\right| \approx C_{d, 5}\left(p_{5,5} v_{5}^{5}+p_{5,3} v_{5}^{3}+p_{5,1} v_{5}\right) .
\end{aligned}
$$

The curve fits (16) and (17) are shown in Figure 3a,b, respectively. Using (15) and (3), we get a Surge-Heave-Pitch model of a pod as,

$$
\dot{\mathbf{X}}=\mathbf{A X}+\mathbf{B}_{\mathbf{p}} \mathrm{F}_{\mathbf{p}}+\mathbf{B}_{\mathbf{v}} \mathbf{F}_{\mathbf{v}}+\mathbf{B}_{\mathbf{e}} \mathrm{F}_{\mathbf{e}}
$$

where,

$$
\begin{gathered}
\mathbf{F}_{\mathbf{p}}=\left[\begin{array}{ll}
F_{p, 5} & F_{p, 3}
\end{array}\right]^{T}, \\
\mathbf{F}_{\mathbf{v}}=\left[\begin{array}{lll}
F_{v, 1} & F_{v, 5} & F_{v, 3}
\end{array}\right]^{T}, \\
\mathbf{F}_{\mathbf{e}}=\left[\begin{array}{lll}
F_{e, 1} & F_{e, 5} & F_{e, 3}
\end{array}\right]^{T},
\end{gathered}
$$




$$
\mathbf{X}=\left[\begin{array}{lllllllllllllll}
v_{1} & v_{5} & x_{5} & \xi_{3} & \xi_{4} & \xi_{5} & \xi_{6} & \xi_{7} & \xi_{8} & \xi_{9} & \xi_{10} & v_{3} & x_{3} & \xi_{1} & \xi_{2}
\end{array}\right]^{T} .
$$

and the radiation force terms are approximated by following state variables using (10),

$$
F_{r, 11}=\xi_{3}, F_{r, 15}=\xi_{5}, F_{r, 51}=\xi_{7}, F_{r, 55}=\xi_{9}, F_{r, 33}=\xi_{1} .
$$

and,

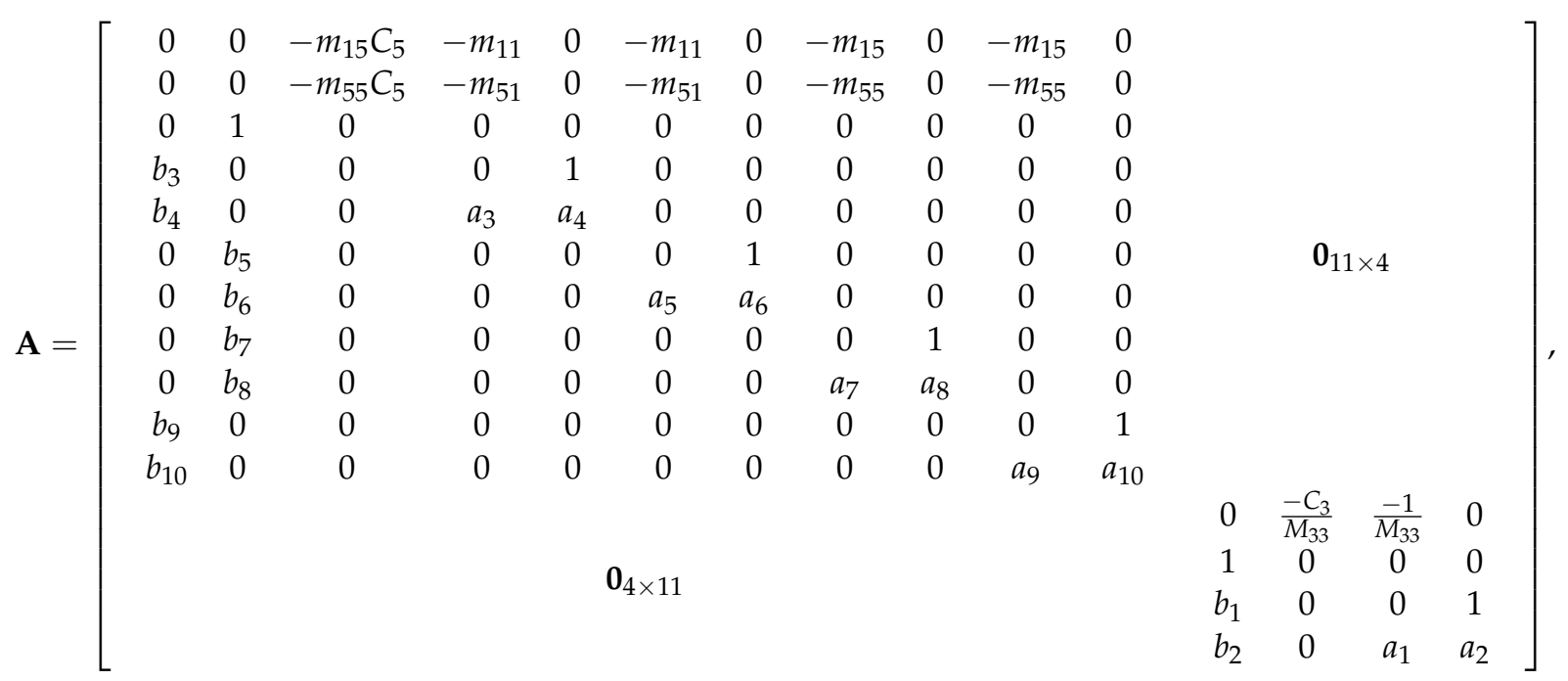

$$
\mathbf{B}_{\mathbf{p}}=\left[\begin{array}{cc}
-m_{15} & 0 \\
-m_{55} & 0 \\
0 & 0 \\
0 & 0 \\
0 & 0 \\
0 & 0 \\
0 & 0 \\
0 & 0 \\
0 & 0 \\
0 & 0 \\
0 & 0 \\
0 & -1 \\
0 & 0 \\
0 & 0 \\
0 & 0
\end{array}\right], \mathbf{B}_{\mathbf{v}}=\left[\begin{array}{ccc}
-m_{11} & -m_{15} & 0 \\
-m_{51} & -m_{55} & 0 \\
0 & 0 & 0 \\
0 & 0 & 0 \\
0 & 0 & 0 \\
0 & 0 & 0 \\
0 & 0 & 0 \\
0 & 0 & 0 \\
0 & 0 & 0 \\
0 & 0 & 0 \\
0 & 0 & 0 \\
0 & 0 & \frac{-1}{M_{33}} \\
0 & 0 & 0 \\
0 & 0 & 0 \\
0 & 0 & 0
\end{array}\right], \mathbf{B}_{\mathbf{e}}=\left[\begin{array}{ccc}
m_{11} & m_{15} & 0 \\
m_{51} & m_{55} & 0 \\
0 & 0 & 0 \\
0 & 0 & 0 \\
0 & 0 & 0 \\
0 & 0 & 0 \\
0 & 0 & 0 \\
0 & 0 & 0 \\
0 & 0 & 0 \\
0 & 0 & 0 \\
0 & 0 & 0 \\
0 & 0 & \frac{1}{M_{33}} \\
0 & 0 & 0 \\
0 & 0 & 0 \\
0 & 0 & 0
\end{array}\right],
$$

Putting (16) and (17) in (18) gives us a 2-DoF (heave and pitch) WEC nonlinear plant model, where the surge is coupled with the pitch and heave is a decoupled DoF. We can use this plant model as a prediction model in NMPC.

\subsection{Nonquadratic WEC-PTO Model}

The electrical power output from the PTO mechanism of the WEC is the difference between the mechanical power input from the waves and the losses in the PTO system. For a given PTO generator, the electrical PTO power cost functional to be maximized, including the electrical losses, is given by,

$$
\max _{F_{p, i}} P_{E, i}=\eta_{\text {Conv }}\left(P_{\text {Mechanical }, i}-P_{\text {Loss }, i}\right)=\eta_{\text {Conv }}\left(F_{p, i} v_{i}-K_{C u}\left[I_{p, i}\left(F_{p, i}\right)\right]^{2} R_{\Omega}\right),
$$

The case study scenario is taken from McCleer Power's Linear PTO generator [3] with the PTO force-current characteristics given by Figure 4 . We can approximate the experimental data in Figure 4 with a mathematical relation, such as a piecewise linear function or a nonlinear function. We have used polynomial approximation which is a 
smooth function. This relation is described by a third-order curve fit between the PTO current and the PTO force,

$$
I_{p, i}\left(F_{p, i}\right)=a_{3, i} F_{p, i}^{3}+a_{2, i} F_{p, i}^{2}+a_{1, i} F_{p, i}+a_{0, i}
$$

Putting (27) in (16), we get,

$$
P_{E, i}=c_{0, i} F_{p, i} v_{i}-\left(c_{1, i} F_{p, i}^{6}+c_{2, i} F_{p, i}^{5}+c_{3, i} F_{p, i}^{4}+c_{4, i} F_{p, i}^{3}+c_{5, i} F_{p, i}^{2}+c_{6, i} F_{p, i}+c_{7, i}\right)
$$

The PTO cost functional surface in (28) is plotted in the PTO velocity-force plane, as shown in Figure 5. The surface plot of the mechanical PTO power, $P_{\text {Mechanical }, i}=F_{p, i} v_{i}$ is non-convex, as shown in Figure 5. However, the electrical PTO power surface, $P_{E, i}$ in (26) has a quadratic power loss term, and it gives convexity to the electrical power surface along the PTO force axis in Figure 5.

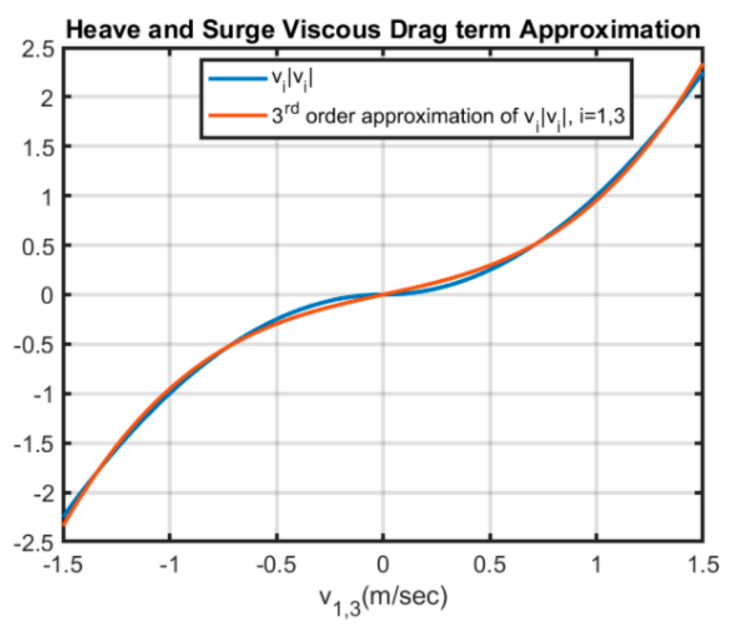

(a)

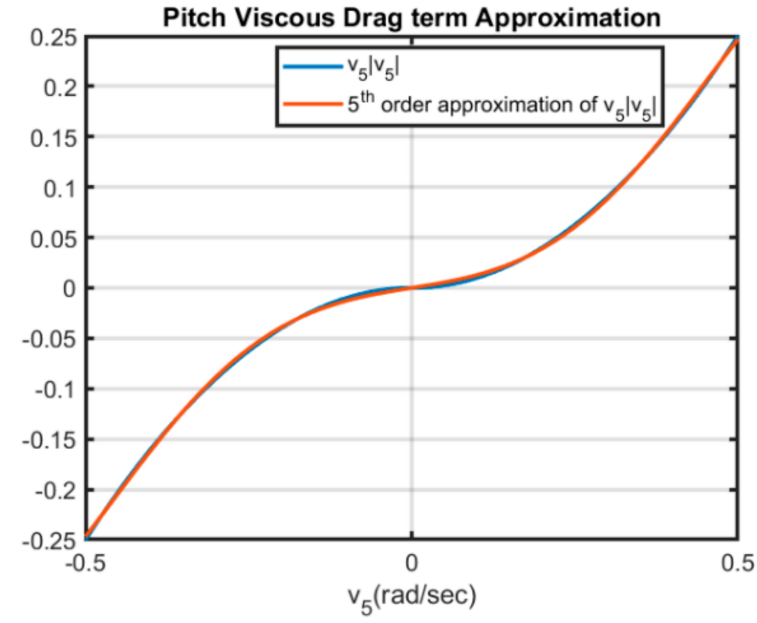

(b)

Figure 3. Polynomial approximations of the quadratic drag term $v_{i}\left|v_{i}\right|:$ (a) 3rd order curve fit for heave and surge axes; (b) 5 th order curve fit for pitch axis.

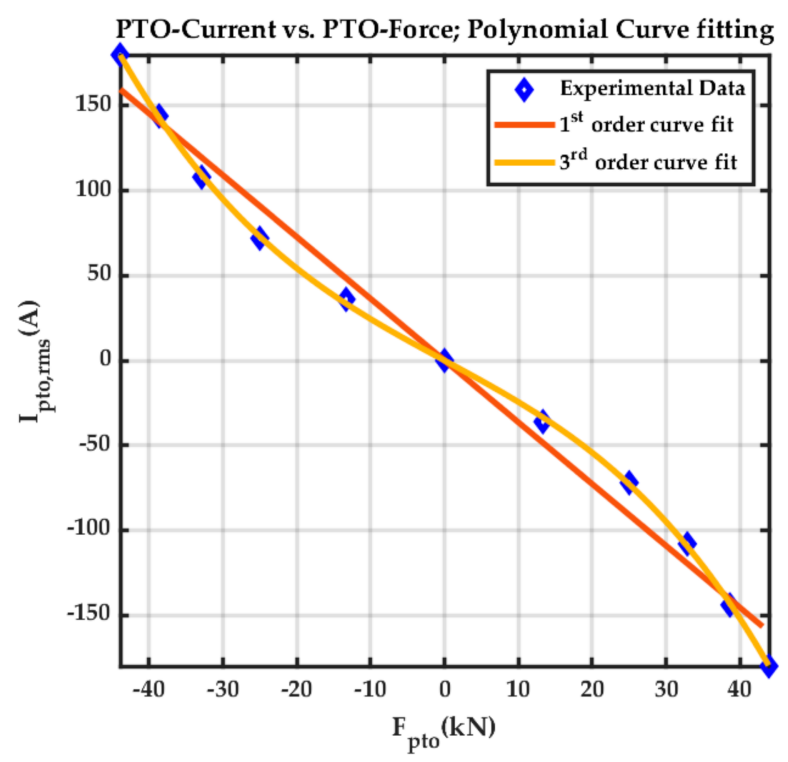

Figure 4. Polynomial curve fitting to the PTO force-current experimental data for a PTO generator. 


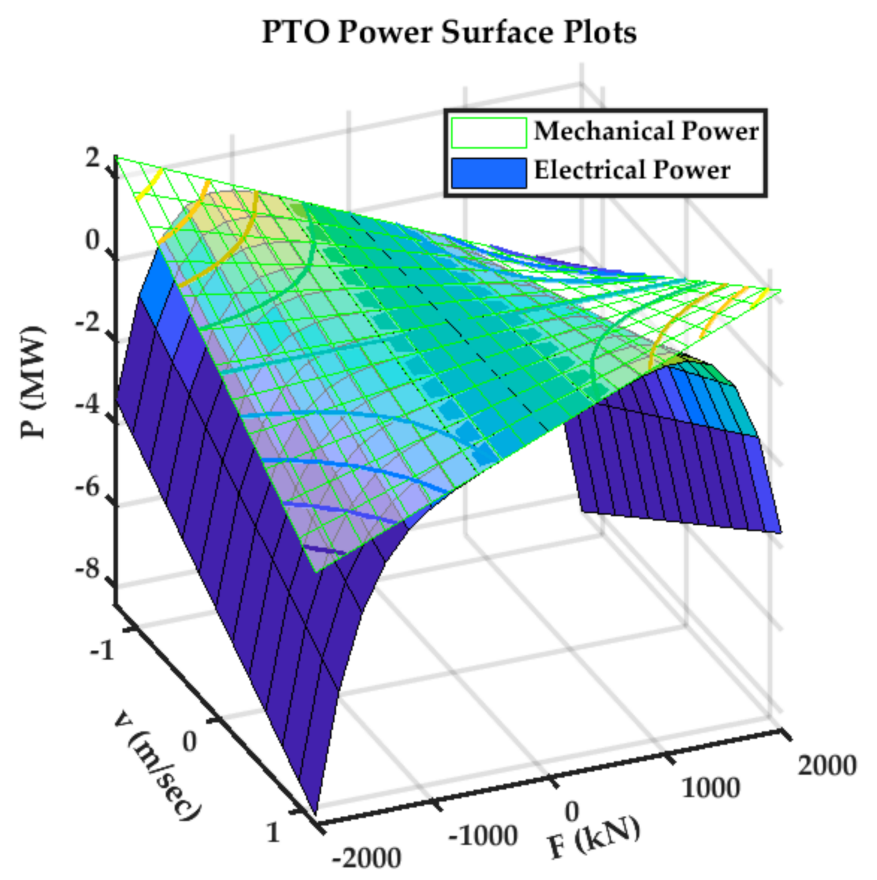

Figure 5. Mechanical and electrical PTO power surface plot in PTO velocity-force plane.

\section{Implementation of NMPC for 2-DoF Heave-Pitch WEC}

The optimal control problem of a WEC involves manipulating the PTO force/torque to maximize the power capture while respecting some system constraints. Various optimal control approaches have been developed, and a comprehensive review can be found in [34]. MPC is a model-based online optimal control solution, and a given NMPC problem optimizes a manipulated variable $\mathrm{u}(t)$ to maximize some cost functional $\mathrm{P}(\cdot)$ while respecting the system constraints. A special class of NMPC problems has been formulated in [30], in which the cost functional takes on a nonlinear piecewise polynomial form. Considering the case of finite horizon optimization control, we can mathematically describe the NMPC problem of such a class as,

$$
\begin{gathered}
\underset{\mathbf{u}(t)}{\operatorname{maximize} \mathbf{P}[t, \dot{\mathbf{X}}(t), \mathbf{X}(t), \mathbf{U}(t), \mathbf{p}(t)]} \\
\text { Where }: \mathbf{P}(\cdot)=\left\{\begin{array}{cc}
P_{1}(\cdot)+\rho_{N, 1}(\cdot), & q_{k}(t)<R_{1} \\
P_{2}(\cdot)+\rho_{N, 2}(\cdot), & R_{1} \leq q_{k}(t) \leq R_{2} \\
\vdots & \vdots \\
P_{j}(\cdot)+\rho_{N, j}(\cdot), & R_{j-1} \leq q_{k}(t) \leq R_{j}
\end{array},\right.
\end{gathered}
$$

subject to,

$$
\begin{gathered}
\text { Dynamic Constraints : } \mathbf{0}=\mathbf{g}(t, \dot{\mathbf{X}}(t), \mathbf{X}(t), \mathbf{U}(t), \mathbf{d}(t), \mathbf{p}(t), N), \\
\text { Boundary Constraint Function : } \mathbf{0}=\mathbf{r}(N, \mathbf{X}(0), \mathbf{U}(0), \mathbf{X}(N), \mathbf{U}(N), \mathbf{p}), \\
\text { Path Constraints Function : } 0 \geq \mathbf{s}(t, \mathbf{X}(t), \mathbf{U}(t), \mathbf{p}(t)) .
\end{gathered}
$$

The description of various variables and constants in (298) through (33) is given in Table 2. The wave excitation force $\mathbf{F}_{\mathbf{e}}$ acting on the hull is considered an unmeasured system disturbance, and based on the available measurements, the controller internally estimates $\mathbf{F}_{\mathbf{e}}$. 
Table 2. Symbols and notations for NMPC formulation.

\begin{tabular}{cc}
\hline Variable & Description \\
\hline$N$ & Prediction horizon \\
$\mathbf{X}$ & State vector \\
$\rho_{N, i}$ & Finite horizon terminal cost penalty or Mayer terms \\
$P_{i}(\cdot)$ & Some Nonlinear functions or Lagrange terms \\
$\mathbf{p}$ & A column vector of time-varying parameters \\
$\mathbf{U}$ & PTO Force manipulated variable vector, $\mathbf{F}_{\mathbf{p}}(N)$ \\
$\mathbf{d}$ & Excitation force disturbance vector, $\mathbf{F}_{\mathbf{e}}(N)$ \\
$q_{k}(t)$ & Cost functional scheduling variable \\
$R_{i}$ & Some real numbers, such that $R_{k+1}>R_{k}$ \\
\hline
\end{tabular}

For the 2-DoF (heave-pitch) WEC problem, the objective function to be maximized in (28) will be the sum of electrical PTO power output in the heave and pitch DoFs for each pod,

$$
P_{E}=P_{E, 3}+P_{E, 5}
$$

Using the technique developed in [30], we can put (34) into the pseudo-quadratic form by defining a suitable $\mathbf{h}_{\mathbf{i}}$ vector for heave and pitch as,

$$
\mathbf{h}_{i}=\left[\begin{array}{lllll}
F_{p, i}^{3} & F_{p, i}^{2} & F_{p, i} & v_{i} & 1
\end{array}\right]^{T}, i=3,5
$$

with,

$$
\mathbf{h}=\left[\begin{array}{l}
\mathbf{h}_{3} \\
\mathbf{h}_{5}
\end{array}\right],
$$

we can reformulate (34) as,

$$
P_{E}=\frac{1}{2} \mathbf{h}^{\mathbf{T}}\left(2\left[\begin{array}{cc}
\mathbf{W}_{3} & \mathbf{0} \\
\mathbf{0} & \mathbf{W}_{\mathbf{5}}
\end{array}\right]\right) \mathbf{h}=\frac{1}{2} \mathbf{h}^{\mathbf{T}}(2 \mathbf{W}) \mathbf{h},
$$

By using (28) in (34), the weighting matrix $\mathbf{W}$ can be obtained by polynomial decomposition of (34) by the vector $\mathbf{h}$ in (36) as the basis vector,

$$
\mathbf{W}_{\mathbf{i}}=\frac{1}{2}\left[\begin{array}{ccccc}
-2 c_{1, i} & -c_{2, i} & 0 & 0 & 0 \\
-c_{2, i} & -2 c_{3, i} & -c_{4, i} & 0 & 0 \\
0 & -c_{4, i} & -2 c_{5, i} & c_{0, i} & -c_{6, i} \\
0 & 0 & c_{0, i} & 0 & 0 \\
0 & 0 & -c_{6, i} & 0 & -2 c_{7, i}
\end{array}\right], i=3,5
$$

The controller is implemented using an ACADO toolkit [21] following the approach developed in [3].

\section{Results}

The schematic diagram of the test setup is shown in Figure 6. The corresponding hardware setup is shown in Figure 7. NMPC is designed in the host machine, which generated code and deployed the controller to the Speedgoat performance real-time target machine [31], model-109100 with Intel Core i3 3.3 GHz, two cores, and 2048 MB DDR3 RAM. The Speedgoat machine is interfaced with a real-time WEC emulator machine through an Ethernet universal data port (UDP) channel. The three WEC pods in Figure 1 are assumed identical, and the same controller is implemented for each pod as shown in Figure 8, while the cross-coupling between pods is ignored for this work. The physical velocity and force constraints of the PTO mechanisms imposed as $\left|v_{3}\right| \leq 2 \mathrm{~m} / \mathrm{sec},\left|v_{5}\right| \leq 0.5 \mathrm{rad} / \mathrm{sec}$ and $\left|F_{p, i}\right| \leq 400 \mathrm{kN}$. The emulated WEC-Sim model of CENTIPOD device is shown in Figure 9. 


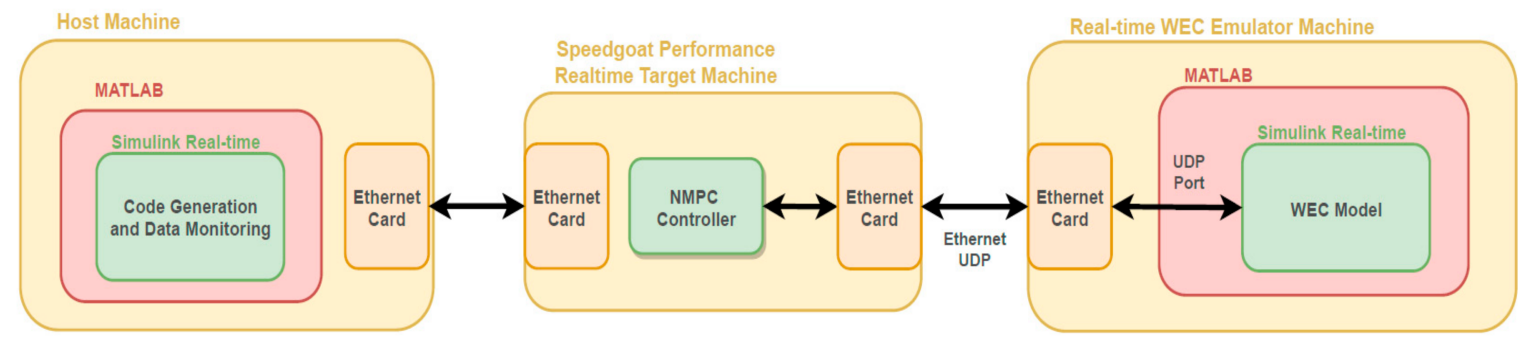

Figure 6. Schematic diagram of the test setup.

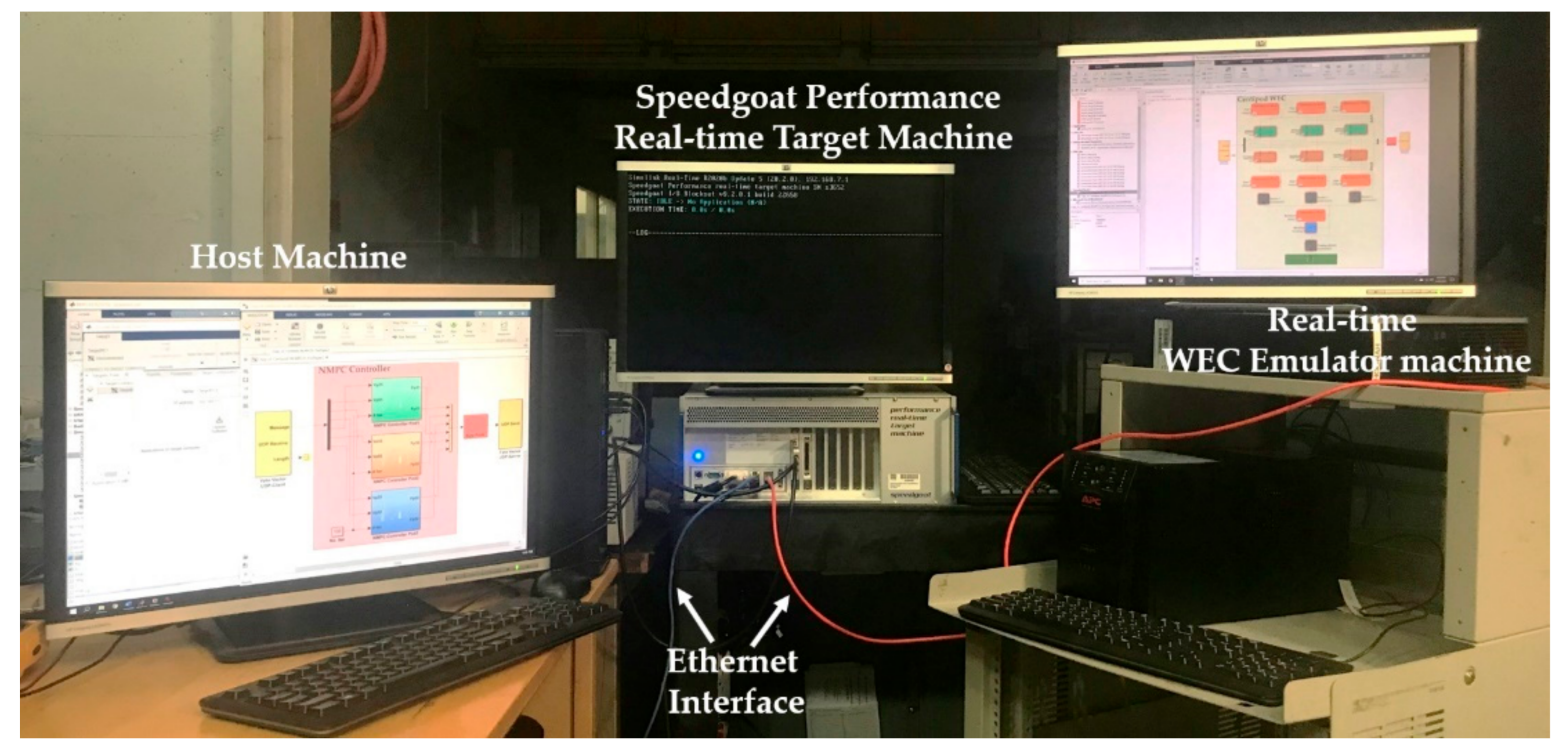

Figure 7. Hardware test setup.

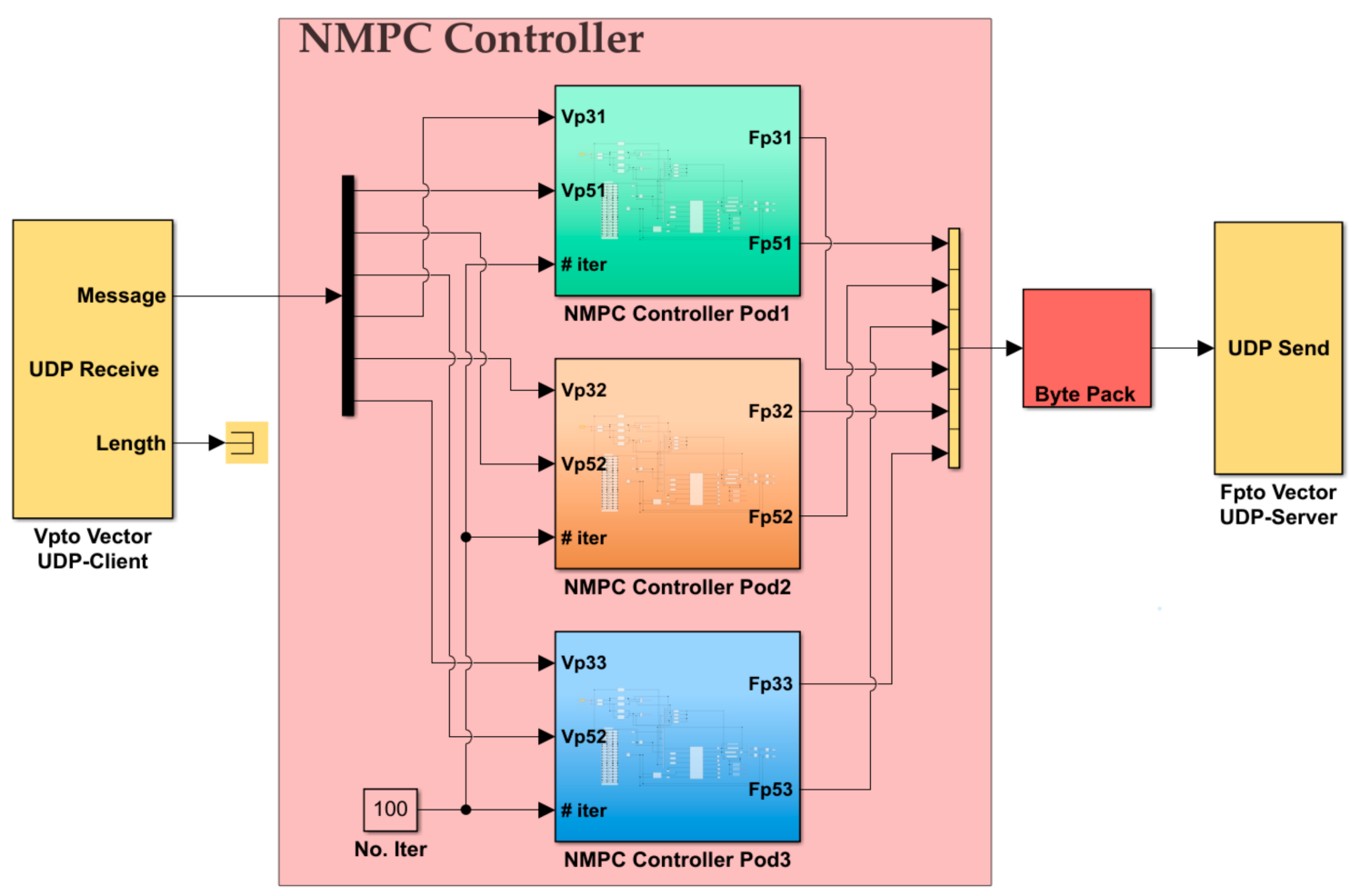

Figure 8. NMPC controller for 2-DoF 3-pod CENTIPOD WEC. 


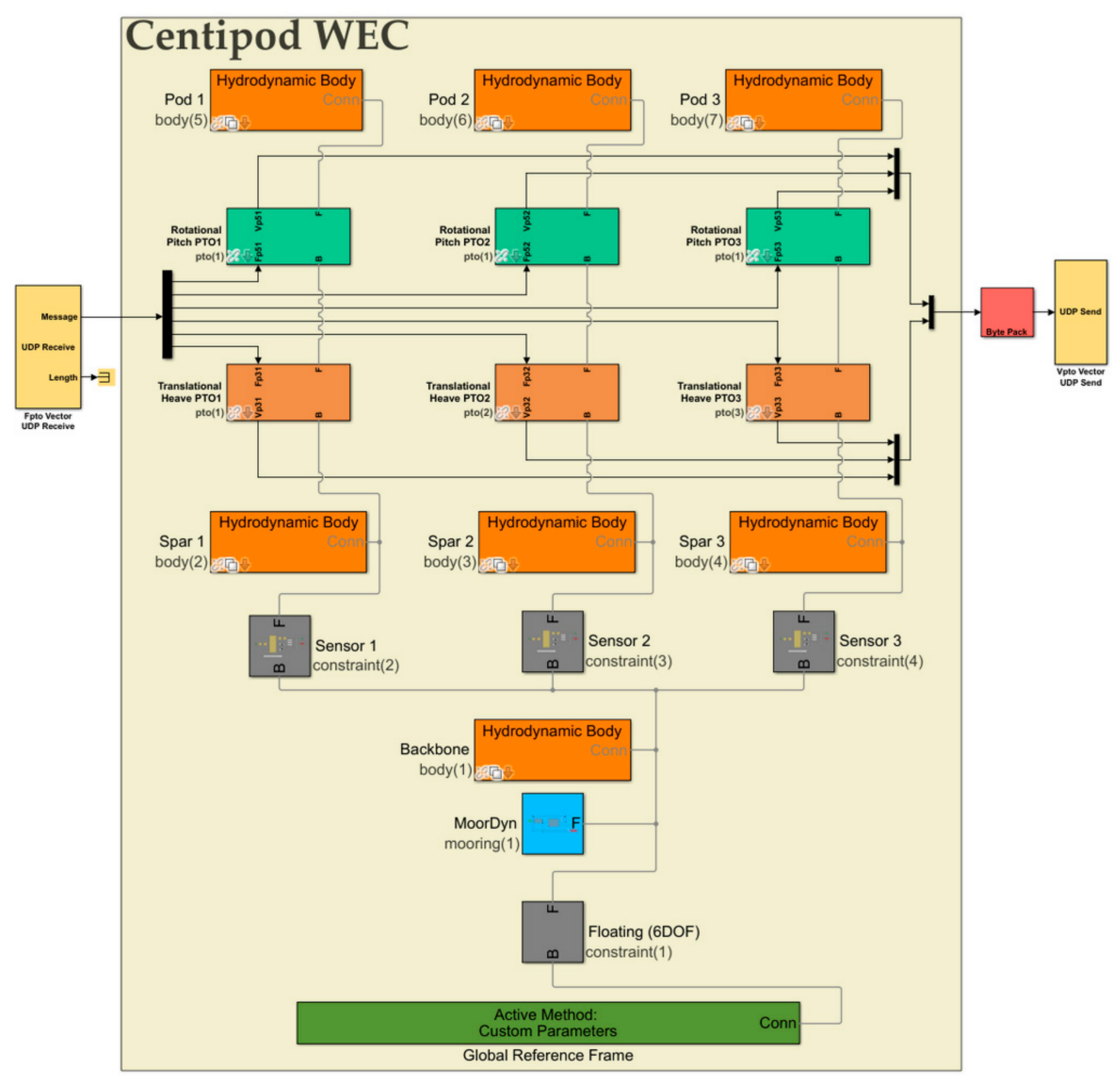

Figure 9. WEC-Sim model of Dehlsen's 2-DoF CENTIPOD device with heave and pitch PTOs for each pod.

Since the WEC pods are assumed identical with no cross-coupling, results are presented only for a single pod. The sea state of interest for WEC-Sim is given in Table 3 . This particular sea state's selection is based on the future testing site of interest for the WEC device, although the hardware testing and a more elaborated study involving other sea states are planned for the future. A step time of $0.1 \mathrm{sec}$ is used for MPC formulation, close to one-tenth of the peak wave period. The performance of NMPC is compared against the linear MPC, and the analysis is performed for the linear and nonlinear hydrodynamics sea conditions.

Table 3. Sea states for WEC-Sim simulation.

\begin{tabular}{cc}
\hline WEC-Sim Simulation Parameter & Value \\
\hline Significant Wave Height [m] & 2.5 \\
Peak Period [s] & 8 \\
Wave Spectrum Type & Pierson Moskowitz (PM) \\
Wave Class & Irregular \\
\hline
\end{tabular}


The average electrical power output results for the heave and pitch PTOs for 2-DoF pod-1 are shown in Figures 10a and 10b, respectively, for linear MPC and NMPC subjected to linear hydrodynamic conditions. Here, we consider the exponentially weighted moving average (EWMA) with the forgetting factor set to unity. The instantaneous electrical power output results corresponding to Figure 10 are shown in Figure 11. The PTO force and wave excitation force profiles for 2-DoF Pod-1 with linear and nonlinear MPC under linear hydrodynamic conditions are shown in Figure 12. The PTO velocity and displacement plots for 2-DoF Pod-1 with linear and nonlinear MPC under linear hydrodynamic conditions are shown in Figure 13.

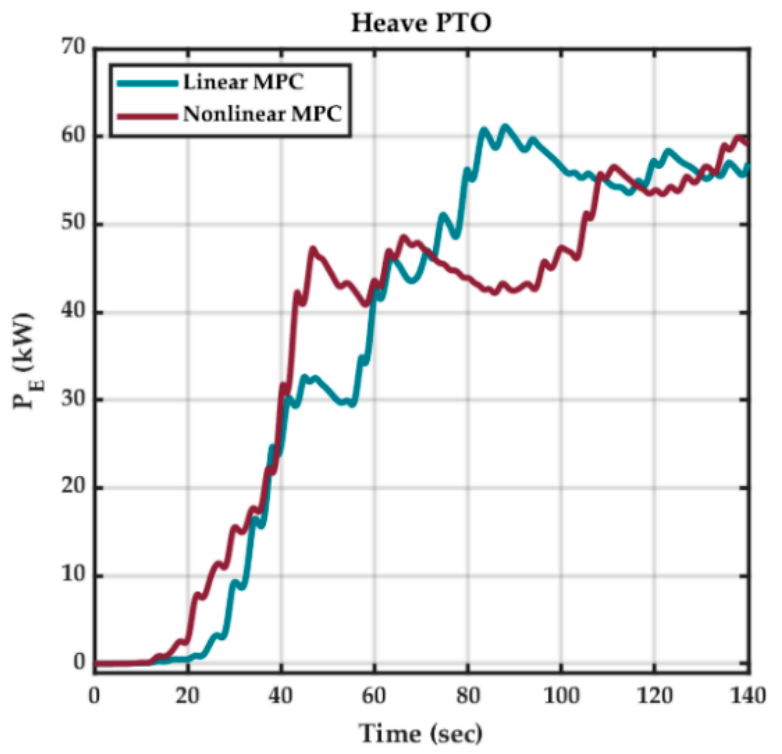

(a)

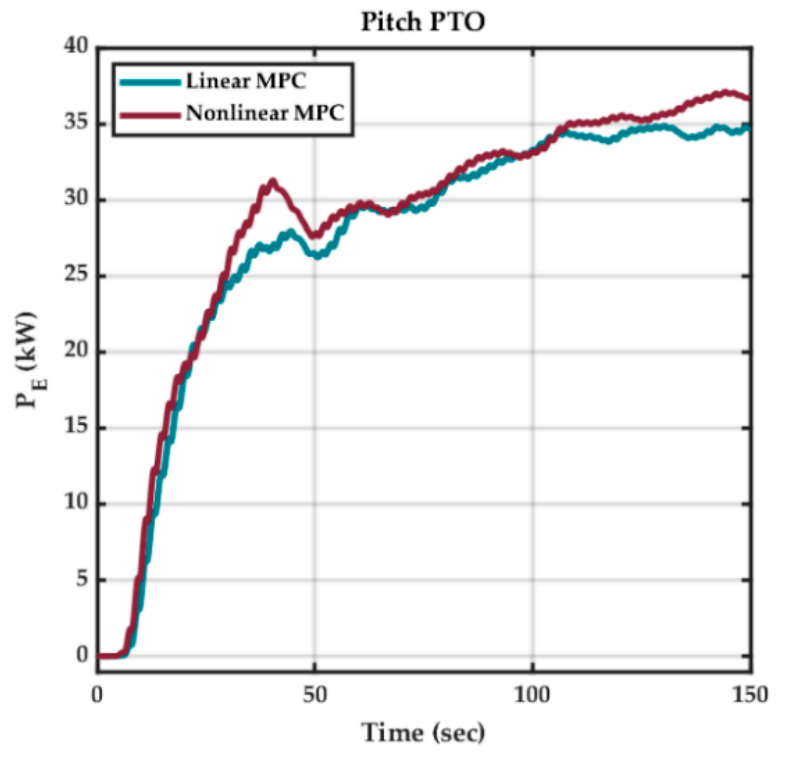

(b)

Figure 10. Average electrical PTO power output for 2-DoF Pod-1 with linear and nonlinear MPC under linear hydrodynamic conditions in WEC-Sim and $\left|F_{p t o}\right| \leq 400 \mathrm{kN}$ : (a) Pod-1 Heave PTO; (b) Pod-1 Pitch PTO.

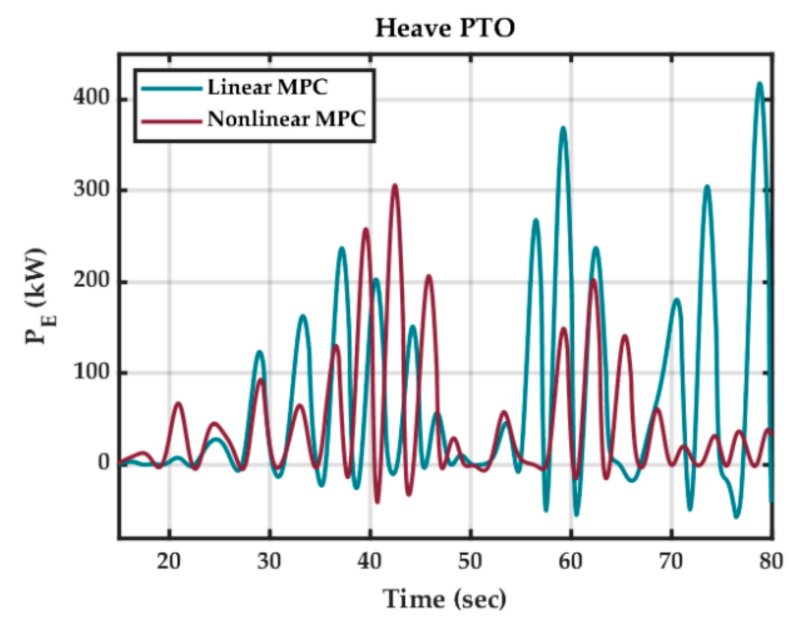

(a)

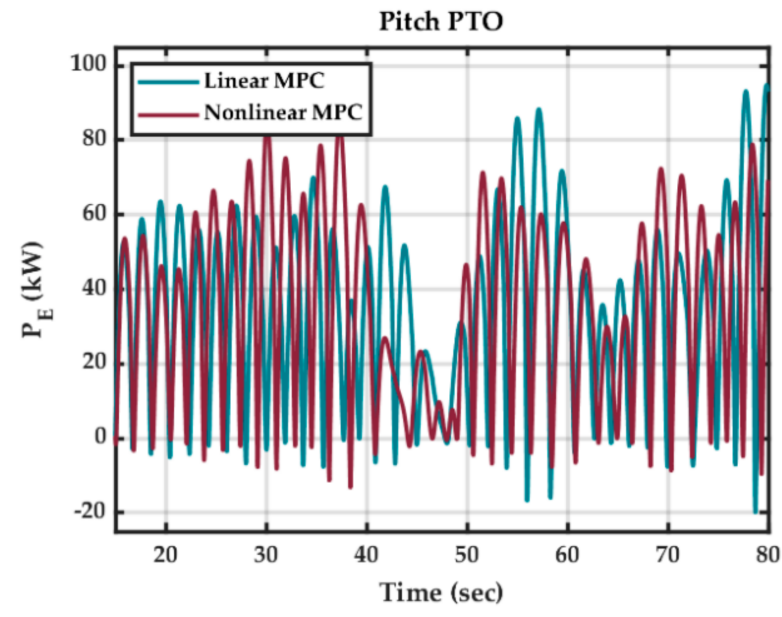

(b)

Figure 11. Instantaneous electrical PTO power output for 2-DoF Pod-1 with linear and nonlinear MPC under linear hydrodynamic conditions in WEC-Sim and $\left|F_{p t o}\right| \leq 400$ kN: (a) Pod-1 Heave PTO; (b) Pod-1 Pitch PTO. 


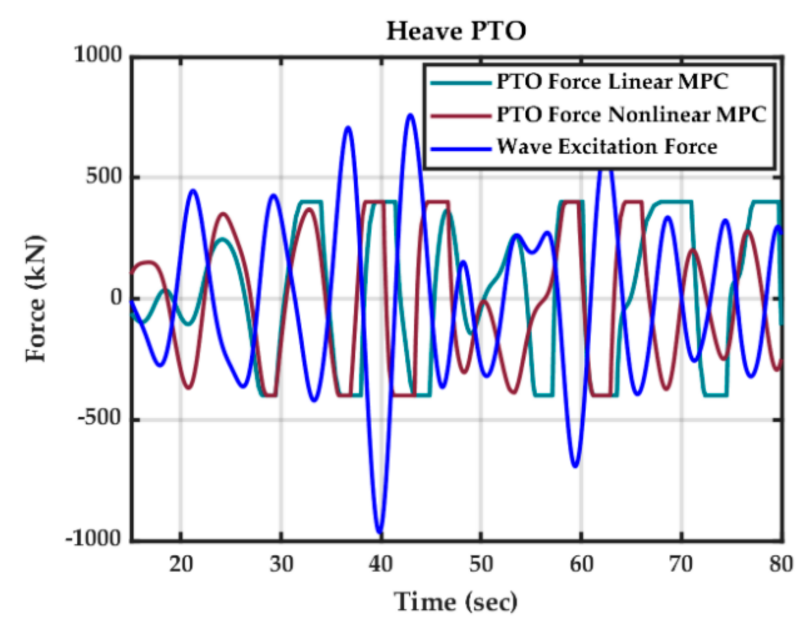

(a)

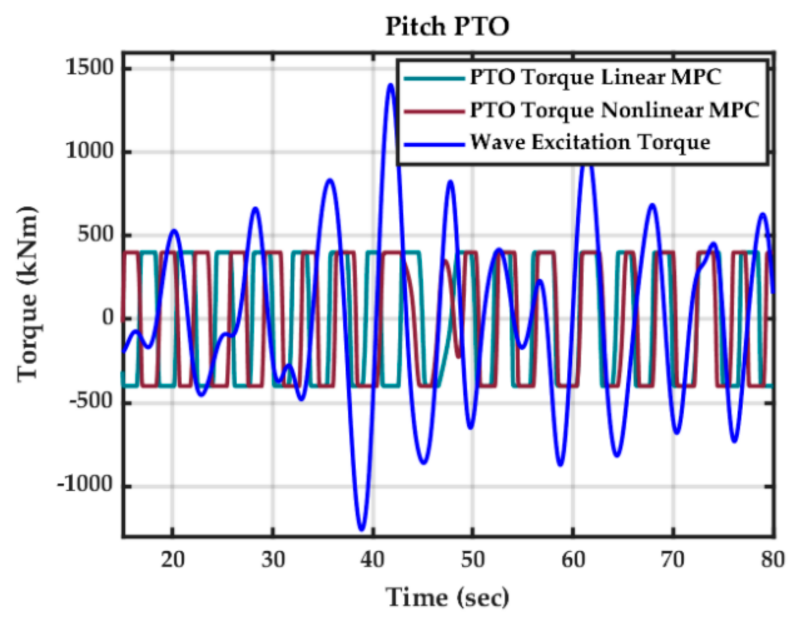

(b)

Figure 12. The PTO force and wave excitation force profiles for 2-DoF Pod-1 with linear and nonlinear MPC under linear hydrodynamic conditions in WEC-Sim and $\left|F_{p t o}\right| \leq 400 k N$ : (a) Pod-1 Heave PTO; (b) Pod-1 Pitch PTO.

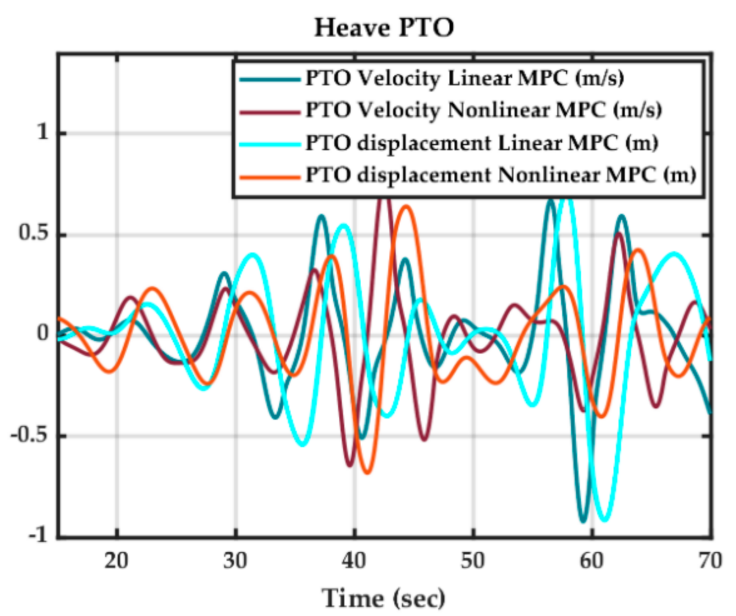

(a)

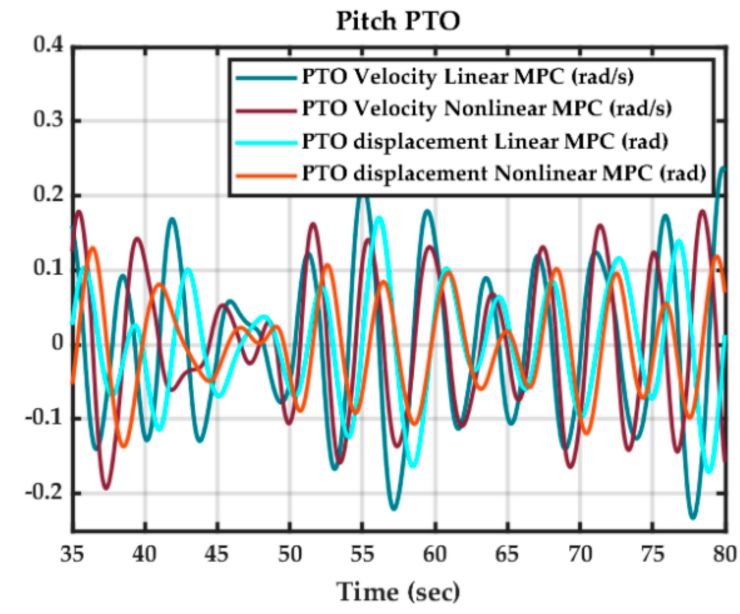

(b)

Figure 13. The PTO velocity and displacement plots for 2-DoF Pod-1 with linear and nonlinear MPC under linear hydrodynamic conditions in WEC-Sim and $\left|F_{p t o}\right| \leq 400 k N$ : (a) Pod-1 Heave PTO; (b) Pod-1 Pitch PTO.

The average and instantaneous electrical power output results under nonlinear hydrodynamics for 2-DoF Pod-1 with linear and nonlinear MPC are shown in Figures 14 and 15, respectively. The comparison of average electrical PTO power output with NMPC for 1-DoF and 2-DoF Pod-1 is shown in Figure 16 under nonlinear hydrodynamic conditions. 


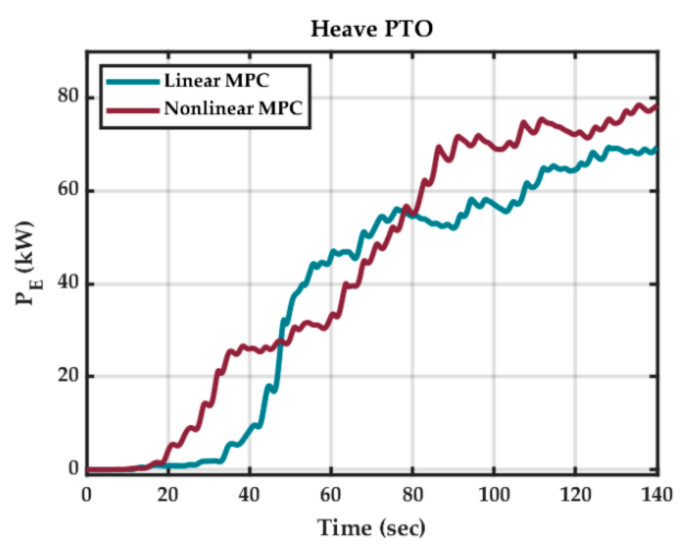

(a)

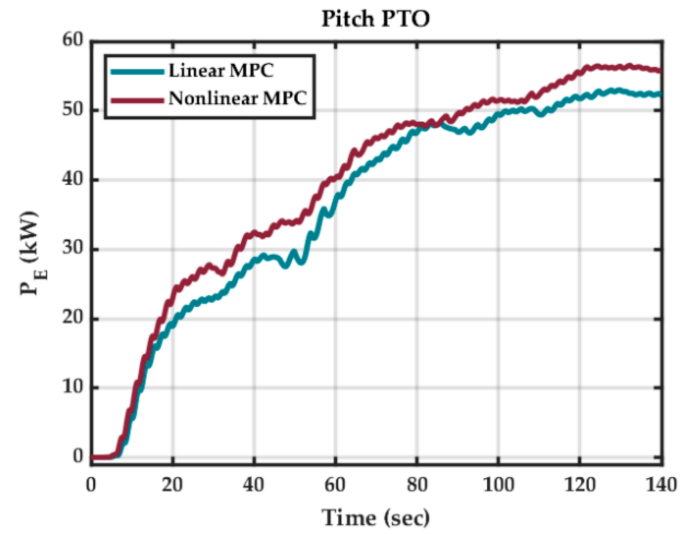

(b)

Figure 14. Average electrical PTO power output for 2-DoF Pod-1 with linear and nonlinear MPC under Nonlinear hydrodynamic conditions in WEC-Sim and $\left|F_{p t o}\right| \leq 400$ kN: (a) Pod-1 Heave PTO; (b) Pod-1 Pitch PTO.

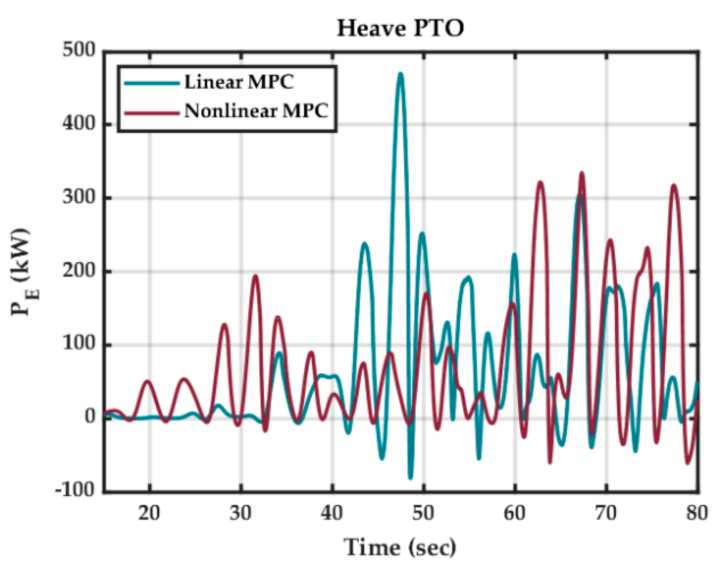

(a)

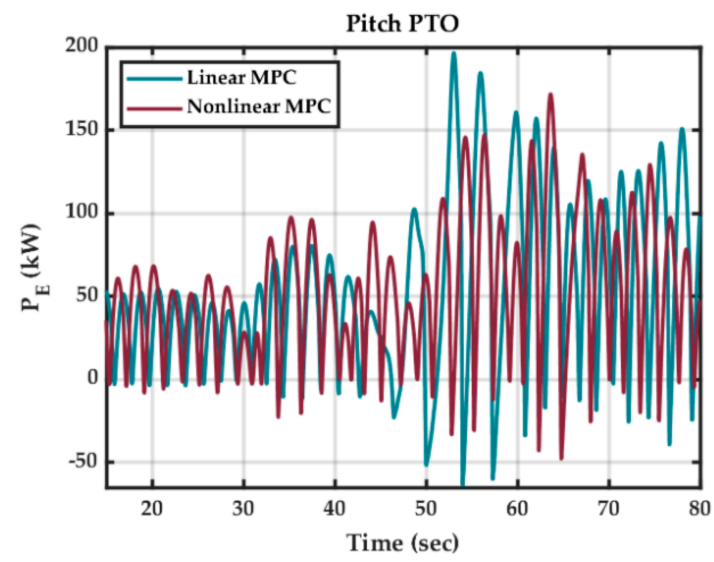

(b)

Figure 15. Instantaneous electrical PTO power output for 2-DoF Pod-1 with linear and nonlinear MPC under nonlinear hydrodynamic conditions in WEC-Sim and $\left|F_{p t o}\right| \leq 400$ kN: (a) Pod-1 Heave PTO; (b) Pod-1 Pitch PTO.

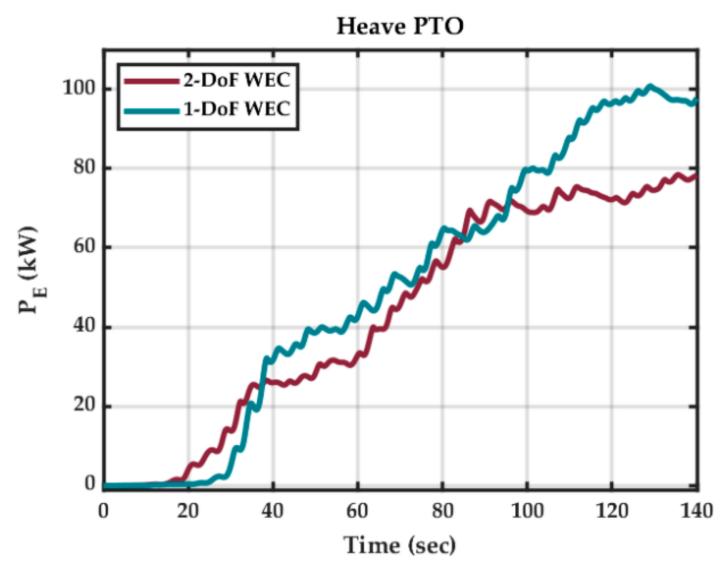

(a)

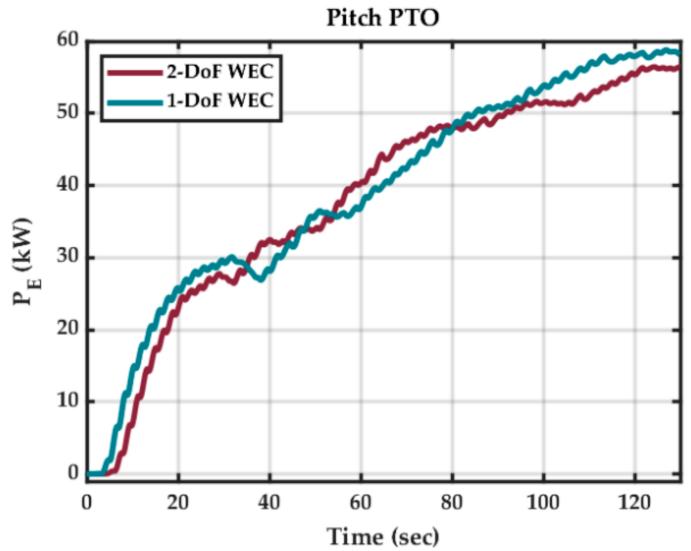

(b)

Figure 16. Average electrical PTO power output for 1-DoF and 2-DoF Pod-1 with nonlinear MPC under nonlinear hydrodynamic conditions in WEC-Sim and $\left|F_{p t o}\right| \leq 400 \mathrm{kN}$ : (a) Pod-1 Heave PTO; (b) Pod-1 Pitch PTO. 


\section{Discussion}

The average electrical power output results in Figures 10 and 14 are summarized in Table 4. NMPC shows a better performance in terms of increased power output when compared to linear MPC. This increase in the output power becomes more prominent under nonlinear hydrodynamic conditions, which are not accounted for by the linear MPC. An overall 5\% increase in power by NMPC compared to linear MPC is obtained under linear hydrodynamic conditions. NMPC obtains an overall $5 \%$ increase in total power output by pod-1 than linear MPC under linear hydrodynamic conditions and $10.6 \%$ under nonlinear hydrodynamic conditions. The corresponding task execution time (TET) stats for the real-time implementations of linear MPC and NMPC in a Speedgoat real-time machine are given in Table 5. Given the controller step time of $0.1 \mathrm{sec}$, the increase in TET for NMPC compared to linear MPC is not very significant.

Table 4. Average electrical power output per PTO for 2-DoF Pod1 with linear MPC and NMPC.

\begin{tabular}{ccccccc}
\hline & \multicolumn{4}{c}{} & \multicolumn{3}{c}{ Average Electrical Power [kW] } \\
\hline \multirow{2}{*}{ Control Algorithm } & \multicolumn{2}{c}{ Linear Hydrodynamic Conditions } & \multicolumn{2}{c}{ Nonlinear Hydrodynamic Conditions } \\
\cline { 2 - 8 } & Heave & Pitch & Total & Heave & Pitch & Total \\
\hline Linear MPC & 57 & 35 & 92 & 70 & 52 & 122 \\
Nonlinear MPC & 60 & 37 & 97 & 79 & 56 & 135 \\
\hline
\end{tabular}

Table 5. Real-time timings stats for Linear MPC vs. Nonlinear MPC.

\begin{tabular}{cccc}
\hline & \multicolumn{3}{c}{ Task Execution Time (TET) [sec] } \\
\hline Control Algorithm & 1-DoF Heave & 1-DoF Pitch & 2-DoF Heave and Pitch \\
\hline Linear MPC & $2.12 \times 10^{-4}$ & $2.67 \times 10^{-4}$ & $5.21 \times 10^{-4}$ \\
Nonlinear MPC & $3.05 \times 10^{-4}$ & $3.21 \times 10^{-4}$ & $6.14 \times 10^{-4}$ \\
\hline
\end{tabular}

The average electrical power output results per PTO for 1-DoF and 2-DoF Pod1 with NMPC from Figure 16 are summarized in Table 6. In moving from 1-DoF WEC to 2-DoF WEC, a $35 \%$ increase in output power is obtained compared to heave only, and $129 \%$ increase compared to pitch only.

Table 6. Average electrical power output per PTO for 1-DoF and 2-DoF Pod1 with NMPC.

\begin{tabular}{ccccc}
\hline & \multicolumn{3}{c}{ Average Electrical Power [kW] } \\
\hline Axis & Heave & 1-DoF WEC & Pitch & 2-DoF WEC \\
Heave and Pitch
\end{tabular}

The locus of electrical PTO power for linear MPC and NMPC under nonlinear hydrodynamic conditions in WEC-Sim, along with the electrical power cost functional surface from Figure 5, are shown in Figure 17.

The locus of electrical PTO power in Figure 17 traverses a trajectory on the cost manifolds and satisfies the cost objective. The cost index formulation in (15) includes a convexifying quadratic term of PTO current, making the resultant electrical PTO surface convex in Figure 5, and with a smooth PTO current profile, the close loop system tends to maintain a stable operation. If the QP problem formulated at a given sample interval is infeasible, the controller will not find a solution. This issue can be handled by monitoring the status of the QP solver during each sampling interval and selecting a suboptimal 
solution when the QP solver fails. An average of 35\% processor load was observed per sampling interval during testing.

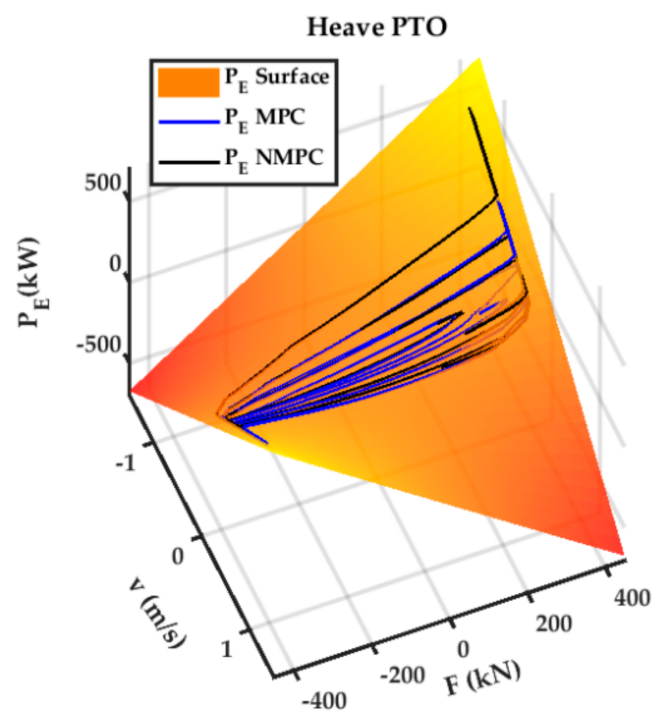

(a)

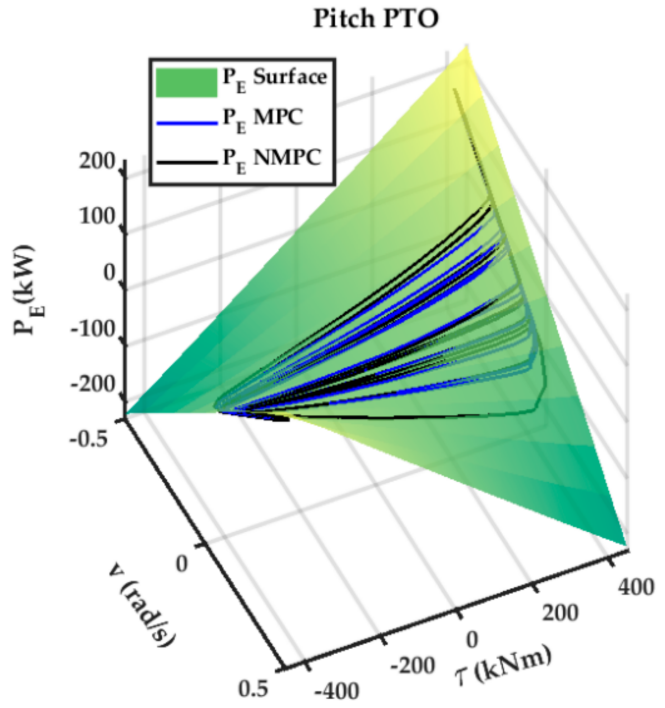

(b)

Figure 17. The locus of electrical PTO power on the electrical power cost functional surface for linear MPC and NMPC under nonlinear hydrodynamic conditions in WEC-Sim and $\left|F_{p t o}\right| \leq 400$ kN: (a) Pod-1 Heave PTO; (b) Pod-1 Pitch PTO.

\section{Conclusions}

This article presents a real-time implementation of NMPC for a nonlinear 2-DoF WEC based on Dehlsen Associates' CENTIPOD multi-pod WEC device, with non-ideal PTOs in the heave and pitch axes. The three pods of the WEC device are assumed identical, and a nonlinear state-space model of a single pod is developed. An NMPC controller is implemented for a 2-DoF WEC device with the cost functional based on a PTO model case study with a highly nonlinear PTO current-force characteristic. The results of the linear MPC are compared with NMPC for the sea states of interest (irregular waves with Pierson Moskowitz spectrum) under linear and nonlinear hydrodynamic conditions in WEC-Sim. The proposed methodology successfully maintained an overall feasible operation of the real-time NMPC problem in simulation as indicated by the status port of the NMPC QP-solver.

An average of 35\% processor load was observed per sampling interval during testing. An overall $5 \%$ increase in total power output by a single pod is obtained by NMPC compared to linear MPC under linear hydrodynamic conditions and $10.6 \%$ under nonlinear hydrodynamic conditions. Moreover, a 35\% increase in net output power is obtained by the 2-DoF WEC device compared to the 1-DoF heave only, and a 129\% increase compared to the 1-DoF pitch only. While the result reflects only a single sea state, the improvement is likely to be reflected similarly in annual energy production (AEP). The AEP would have a substantive impact on the levelized cost of energy (LCOE). The present work did not consider the cross-coupling between the three pods of the CENTIPOD device. The cross-coupling would be investigated in future work with anticipation of a further increase in the captured power for the sea conditions where the cross-coupling effect is no longer negligible. 
Author Contributions: Conceptualization, A.M.; methodology, A.S.H., T.K.A.B. and A.M.; software, A.M. and A.S.H.; validation, A.S.H., T.K.A.B. and A.M.; formal analysis, A.S.H.; investigation, A.S.H., T.K.A.B. and A.M.; resources, T.K.A.B. and A.M.; data curation, A.S.H. and A.M.; writing-A.S.H.; writing-review and editing, T.K.A.B. and A.M.; visualization, A.S.H.; supervision, T.K.A.B.; project administration, A.M. and T.K.A.B.; funding acquisition, A.M. and T.K.A.B. All authors have read and agreed to the published version of the manuscript.

Funding: This work was funded under the TEAMER program by U.S. Department of Energy's Energy Efficiency and Renewable Energy (EERE) office within the Water Power Technologies Office (WPTO).

Institutional Review Board Statement: Not applicable.

Informed Consent Statement: Not applicable.

Acknowledgments: The authors would like to thank Dehlsen Associates, LLC and McCleer Power for their technical support and for providing the experimental data.

Conflicts of Interest: The authors declare no conflict of interest.

\section{References}

1. Muetze, A.; Vining, J.G. Ocean Wave Energy Conversion-A Survey. In Proceedings of the Conference Record of the 2006 IEEE Industry Applications Conference Forty-First IAS Annual Meeting, Tampa, Fl, USA, 8-12 October 2006; Volume 3, pp. $1410-1417$.

2. Richter, M.; Magana, M.E.; Sawodny, O.; Brekken, T.K.A. Nonlinear Model Predictive Control of a Point Absorber Wave Energy Converter. IEEE Trans. Sustain. Energy 2013, 4, 118-126. [CrossRef]

3. Haider, A.S.; Brekken, T.K.A.; McCall, A. A State-of-the-Art Strategy to Implement Nonlinear Model Predictive Controller with Non-Quadratic Piecewise Discontinuous Cost Index for Ocean Wave Energy Systems. In Proceedings of the 2020 IEEE Energy Conversion Congress and Exposition (ECCE), Detroit, MI, USA, 11-15 October 2020; pp. 1873-1878.

4. Brekken, T.K.A. On Model Predictive Control for a Point Absorber Wave Energy Converter. In Proceedings of the 2011 IEEE Trondheim PowerTech, Trondheim, Norway, 19-23 June 2011; pp. 1-8.

5. Faedo, N.; Olaya, S.; Ringwood, J.V. Optimal Control, MPC and MPC-like Algorithms for Wave Energy Systems: An Overview. IFAC J. Syst. Control 2017, 1, 37-56. [CrossRef]

6. Zhong, Q.; Yeung, R.W. Model-Predictive Control Strategy for an Array of Wave-Energy Converters. J. Mar. Sci. Appl. 2019, 18, 26-37. [CrossRef]

7. Genest, R.; Ringwood, J.V. A Critical Comparison of Model-Predictive and Pseudospectral Control for Wave Energy Devices. J. Ocean Eng. Mar. Energy 2016, 2, 485-499. [CrossRef]

8. Li, G.; Belmont, M.R. Model Predictive Control of Sea Wave Energy Converters-Part I: A Convex Approach for the Case of a Single Device. Renew. Energy 2014, 69, 453-463. [CrossRef]

9. Li, G.; Belmont, M.R. Model Predictive Control of Sea Wave Energy Converters-Part II: The Case of an Array of Devices. Renew. Energy 2014, 68, 540-549. [CrossRef]

10. Starrett, M.; So, R.; Brekken, T.K.A.; McCall, A. Increasing Power Capture from Multibody Wave Energy Conversion Systems Using Model Predictive Control. In Proceedings of the 2015 IEEE Conference on Technologies for Sustainability (SusTech), Ogden, UT, USA, 30 July-1 August 2015; pp. 20-26.

11. Falcão, A.F.O.; Henriques, J.C.C. Effect of Non-Ideal Power Take-off Efficiency on Performance of Single- and Two-Body Reactively Controlled Wave Energy Converters. J. Ocean Eng. Mar. Energy 2015, 1, 273-286. [CrossRef]

12. Davis, A.F.; Thomson, J.; Mundon, T.R.; Fabien, B.C. Modeling and Analysis of a Multi Degree of Freedom Point Absorber Wave Energy Converter. In Proceedings of the OMAE 2014, San Francisco, CA, USA, 8 June 2014; Volume 8A: Ocean Engineering.

13. Al Shami, E.; Wang, X.; Ji, X. A Study of the Effects of Increasing the Degrees of Freedom of a Point-Absorber Wave Energy Converter on Its Harvesting Performance. Mech. Syst. Signal Process. 2019, 133, 106281. [CrossRef]

14. Strager, T.; Martin dit Neuville, A.; Fernández López, P.; Giorgio, G.; Mureşan, T.; Andersen, P.; Nielsen, K.M.; Pedersen, T.S.; Vidal Sánchez, E. Optimising Reactive Control in Non-Ideal Efficiency Wave Energy Converters. In Proceedings of the OMAE 2014, San Francisco, CA, USA, 8 June 2014; Volume 9A: Ocean Renewable Energy.

15. Perdigão, J.; Sarmento, A. Overall-Efficiency Optimisation in OWC Devices. Appl. Ocean Res. 2003, 25, 157-166. [CrossRef]

16. Tedeschi, E.; Carraro, M.; Molinas, M.; Mattavelli, P. Effect of Control Strategies and Power Take-Off Efficiency on the Power Capture From Sea Waves. IEEE Trans. Energy Convers. 2011, 26, 1088-1098. [CrossRef]

17. Jia, Y.; Meng, K.; Dong, L.; Liu, T.; Sun, C.; Dong, Z.Y. Economic Model Predictive Control of a Point Absorber Wave Energy Converter. IEEE Trans. Sustain. Energy 2021, 12, 578-586. [CrossRef]

18. Ellis, M.; Durand, H.; Christofides, P.D. A Tutorial Review of Economic Model Predictive Control Methods. J. Process Control 2014, 24, 1156-1178. [CrossRef]

19. Rawlings, J.B.; Angeli, D.; Bates, C.N. Fundamentals of Economic Model Predictive Control. In Proceedings of the 2012 IEEE 51 st IEEE Conference on Decision and Control (CDC), Maui, HI, USA, 10-13 December 2012; pp. 3851-3861. 
20. Houska, B.; Ferreau, H.J.; Diehl, M. An Auto-Generated Real-Time Iteration Algorithm for Nonlinear MPC in the Microsecond Range. Automatica 2011, 47, 2279-2285. [CrossRef]

21. Houska, B.; Ferreau, H.J.; Diehl, M. ACADO Toolkit-An Open-Source Framework for Automatic Control and Dynamic Optimization. Optim. Control. Appl. Methods 2011, 32, 298-312. [CrossRef]

22. Abdelkhalik, O.; Zou, S.; Robinett, R.D.; Bacelli, G.; Wilson, D.G.; Coe, R.; Korde, U. Multiresonant Feedback Control of a Three-Degree-of-Freedom Wave Energy Converter. IEEE Trans. Sustain. Energy 2017, 8, 1518-1527. [CrossRef]

23. Frequency- and Time-Domain Analysis of a Multi-Degree-of-Freedom Point Absorber Wave Energy Converter-Yizhi Ye, Weidong Chen, 2017. Available online: https://journals.sagepub.com/doi/full/10.1177/1687814017722081 (accessed on 20 July 2021).

24. Coe, R.G.; Bull, D.L. Nonlinear Time-Domain Performance Model for a Wave Energy Converter in Three Dimensions. In Proceedings of the 2014 Oceans, St. John's, NL, Canada, 14-19 September 2014; pp. 1-10.

25. Huang, S.; Shi, H.; Dong, X. Capture Performance of A Multi-Freedom Wave Energy Converter with Different Power Take-off Systems. China Ocean Eng. 2019, 33, 288-296. [CrossRef]

26. Hillis, A.J.; Whitlam, C.; Brask, A.; Chapman, J.; Plummer, A.R. Active Control for Multi-Degree-of-Freedom Wave Energy Converters with Load Limiting. Renew. Energy 2020, 159, 1177-1187. [CrossRef]

27. Li, G. Nonlinear Model Predictive Control of a Wave Energy Converter Based on Differential Flatness Parameterisation. Int. J. Control 2017, 90, 68-77. [CrossRef]

28. WEC-Sim (Wave Energy Converter SIMulator) -WEC-Sim Documentation. Available online: https://wec-sim.github.io/WECSim/ (accessed on 27 March 2021).

29. van Rij, J.; Yu, Y.-H.; McCall, A.; Coe, R.G. Extreme Load Computational Fluid Dynamics Analysis and Verification for a Multibody Wave Energy Converter. In Proceedings of the OMAE 2019, Glasgow, UK, 9-14 June 2019; Volume 10: Ocean Renewable Energy.

30. Haider, A.S.; Brekken, T.K.A.; McCall, A. Application of Real-Time Nonlinear Model Predictive Control for Wave Energy Conversion. IET Renew. Power Gener. 2021. [CrossRef]

31. Speedgoat-The Quickest Path to Real-Time Simulation and Testing. Available online: https://www.speedgoat.com/ (accessed on 23 February 2021).

32. Ecomerit Technologies. Available online: http://www.ecomerittech.com/centipod.php (accessed on 14 August 2021).

33. Wamit, Inc. The State of the Art in Wave Interaction Analysis. Available online: https://www.wamit.com/ (accessed on 28 March 2021).

34. Ringwood, J.V. Wave Energy Control: Status and Perspectives $2020 * *$ This Paper Is Based upon Work Supported by Science Foundation Ireland under Grant No. 13/IA/1886 and Grant No. 12/RC/2302 for the Marine Renewable Ireland (MaREI) Centre. IFAC-PapersOnLine 2020, 53, 12271-12282. [CrossRef] 